\title{
Oct4 transcriptionally regulates the expression of long non-coding RNAs NEAT1 and MALAT1 to promote lung cancer progression
}

Jayu Jen ${ }^{1,2}$, Yen-An Tang ${ }^{1,2}$, Ying-Hung Lu', Che-Chung Lin ${ }^{1}$, Wu-Wei Lai ${ }^{3}$ and Yi-Ching Wang ${ }^{1,2^{*}}$ (D)

\begin{abstract}
Background: Oct4, a key stemness transcription factor, is overexpressed in lung cancer. Here, we reveal a novel transcription regulation of long non-coding RNAs (IncRNAs) by Oct4. LncRNAs have emerged as important players in cancer progression.

Methods: Oct4 chromatin-immunoprecipitation (ChIP)-sequencing and several IncRNA databases with literature annotation were integrated to identify Oct4-regulated IncRNAs. Luciferase activity, GRT-PCR and ChIP-PCR assays were conducted to examine transcription regulation of IncRNAs by Oct4. Reconstitution experiments of Oct4 and downstream IncRNAs in cell proliferation, migration and invasion assays were performed to confirm the Oct4-IncRNAs signaling axes in promoting lung cancer cell growth and motility. The expression correlations between Oct4 and IncRNAs were investigated in 124 lung cancer patients using qRT-PCR analysis. The clinical significance of Oct4/IncRNAs signaling axes were further evaluated using multivariate Cox regression and Kaplan-Meier analyses.

Results: We confirmed that seven IncRNAs were upregulated by direct binding of Oct4. Among them, nuclear paraspeckle assembly transcript 1 (NEAT1), metastasis-associated lung adenocarcinoma transcript 1 (MALAT1) and urothelial carcinoma-associated 1 (UCA1) were validated as Oct4 transcriptional targets through promoter or enhancer activation. We showed that lung cancer cells overexpressing NEAT1 or MALAT1 and the Oct4-silenced cells reconstituted with NEAT1 or MALAT1 promoted cell proliferation, migration and invasion. In addition, knockdown of NEAT1 or MALAT1 abolished Oct4-mediated lung cancer cell growth and motility. These cell-based results suggested that Oct4/NEAT1 or Oct4/MALAT1 axis promoted oncogenesis. Clinically, Oct4/NEAT1/MALAT1 co-overexpression was an independent factor for prediction of poor outcome in 124 lung cancer patients.

Conclusions: Our study reveals a novel mechanism by which Oct4 transcriptionally activates NEAT1 via promoter and MALAT1 via enhancer binding to promote cell proliferation and motility, and led to lung tumorigenesis and poor prognosis.
\end{abstract}

Keywords: Oct4, IncRNA, MALAT1, NEAT1, Transcription regulation, Lung cancer

\footnotetext{
* Correspondence: ycw5798@mail.ncku.edu.tw

'Department of Pharmacology, College of Medicine, National Cheng Kung

University, Tainan 701, Taiwan

${ }^{2}$ Institute of Basic Medical Sciences, College of Medicine, National Cheng

Kung University, Tainan 701, Taiwan

Full list of author information is available at the end of the article
} 


\section{Background}

Oct4, encoded by POU5F1 (POU domain, class 5, transcription factor 1), is a homeodomain transcription factor of the POU family. Oct4, Sox2 and Nanog are well-known pluripotency-associated transcription factors which maintain embryonic stem cells state [1]. Metaplastic transformation, a precancerous condition, has been reported to recapitulate embryonic development. Therefore, the key factors involved in embryonic development may play critical roles in carcinogenesis. Studies have shown that Oct4 is overexpressed in human cancers such as bladder [2], breast [3], cervical cancer [4], oral squamous cell carcinoma [5], hepatocellular carcinoma [6] and lung cancer [7, 8]. In embryonic stem cells, Oct4 has been identified to regulate transcriptions of other transcription factors, chromatin modifiers, long non-coding RNAs (lncRNAs) and microRNAs [9, 10]. For instance, Oct4 regulates lncRNAs expression, such as linc-RoR, which is a key reprogramming factor associated with pluripotency [11]. Oct4 can also interact with Pontin, a chromatin remodeling factor, to regulate the transcription of lncRNAs, including linc1253, a lineage programme repressing lincRNA [12]. However, transcription regulation of IncRNAs by Oct4 in tumorigenesis remains elusive.

LncRNAs is a subset of non-coding RNAs with length ranging from 200 nucleotides to 100,000 nucleotides. According to data obtained using next generation RNAsequencing, the number of total human lncRNAs is approximately 20,000 transcripts and over 200 lncRNAs are confirmed to be functional $[13,14]$. Some lncRNAs are dysregulated in cancers and may serve as potential prognostic markers for specific cancer types $[15,16]$. Some lncRNAs have been characterized to possess oncogene-like or tumor suppressor-like function. For instance, Hox Antisense Intergenic RNA (HOTAIR), acts as a bridge between PRC2 chromatin repressive and LSD1/CoREST/ REST corepressor complexes to further modulate the metastasis-related gene expressions through changing chromatin states in breast cancer [15, 17]. Another IncRNA, HOXA transcript at the distal tip (HOTTIP), not only promotes pancreatic cancer progression but also confers chemoresistance to gemcitabine, which may be mediated by HOXA13 [18, 19]. Accumulating evidence indicates that IncRNAs play critical roles in cancer biology.

Up to date, most of the studies on lncRNAs focus on the outcome and underlying mechanisms of dysregulated lncRNAs and their potential as prognosis markers. However, little is known about the upstream regulations responsible for aberrant expression of lncRNAs in cancers, especially at the transcriptional level. Our previous study using chromatin-immunoprecipitation sequencing (ChIP-seq) and functional analyses revealed a critical
Oct4-driven transcriptional program [8]. Genome-wide analysis of Oct4 targeting of this program suggests a novel role of Oct4-mediated transcriptional regulation of lncRNAs. In the current study, we have shown that Oct4 transcriptionally activated oncogenic lncRNAs expression through promoter- or enhancer-binding regulation. Moreover, Oct4-mediated high expression of lncRNAs such as nuclear paraspeckle assembly transcript 1 (NEAT1) and metastasis-associated lung adenocarcinoma transcript 1 (MALAT1) promoted lung cancer cell proliferation, migration and invasion abilities. Clinical studies further validated the importance of Oct4/NEAT1/ $M A L A T 1$ signaling axis in lung cancer progression.

\section{Methods}

Cell lines and culture conditions

Human lung adenocarcinoma cell line A549 and normal bronchial epithelial cell line BEAS-2B was purchased from American Tissue Culture Company (ATCC). Human lung adenocarcinoma cell line CL1-0 was obtained from Dr. Pan-Chyr Yang (Department of Internal Medicine Medical College, National Taiwan University, Taiwan). All media were supplemented with $10 \%$ Fetal Bovine Serum (Gibco, Carlsbad, CA, USA) and 1\% penicillin/streptomycin (Gibco). Stable cell line expressing Oct4 or empty vector was established by ectopic transfection of Flag-Oct4 or empty vector plasmid into A549 and CL1-0 cells, and selected with puromycin. Transient transfections of Oct4 in BEAS-2B were carried out with lipofetamine 2000 (Invitrogen, Carslbad, CA, USA).

\section{Transfection of plasmids and RNAi}

The plasmids used in the study are listed in Additional file 1: Table S1. The interference RNA (RNAi) for Oct4 was obtained from Invitrogen (\# Oct4-HSS143403, Invitrogen). Depletion of NEAT1 or MALAT1 was performed by transfection of smart-pool siRNAs (Dharmacon, Lafayette, CO, USA) at final concentration of $10 \mathrm{nM}$. Transfections of expression plasmids and RNAi were performed using lipofectamine 2000 (Invitrogen, Carslbad, CA, USA) according to the manufacturer's protocol.

\section{Chromatin-immunoprecipitation-polymerase chain reaction (ChIP-PCR) assay}

Empty vector control and Oct4 stably-overexpressed A549 cells $\left(1 \times 10^{7}\right.$ cells $)$ were cross-linked with $1 \%$ formaldehyde for $10 \mathrm{~min}$ at $37{ }^{\circ} \mathrm{C}$, followed by preparation of nuclear lysates using Magna $\mathrm{ChIP}^{\mathrm{mm}}$ protein G Kit (Millipore Co., Billerica, MA, USA). Nuclear lysates were sonicated to shear crosslinked DNA to around $300 \sim 500$ bps using Covaris-S2 machine. Chromatin was immunoprecipitated with Oct4 antibody (1:100, \# ab19857, Abcam, Cambridge, UK). Purified chromatinimmunoprecipitated DNA was subjected to PCR analysis 
using primers for the lncRNA promoter and enhancer regions listed in Additional file 1: Table S2.

\section{RNA extraction and quantitative reverse transcriptase-} polymerase chain reaction (qRT-PCR) assays

Four $\mu \mathrm{g}$ of total RNA was reverse transcribed to cDNA using MultiScribe ${ }^{\text {TM }}$ reverse transcriptase (Applied Biosystems, Foster City, CA, USA). cDNA was amplified using the Fast SYBR ${ }^{\bullet}$ Green Master Mix (Applied Biosystems). qRT-PCR was used to measure Oct4 mRNA and lncRNA expression using the StepOnePlus ${ }^{\mathrm{Ta}}$ RealTime PCR System (Applied Biosystems). The primer sequences and annealing temperature are listed in Additional file 1: Table S3.

\section{Site-directed mutagenesis and luciferase promoter/enhancer activity assays}

Mutations (Mut) of Oct4 binding elements within the NEAT1 promoter, MALAT1 enhancer or urothelial carcinoma-associated 1 (UCA1) enhancer were generated by site-directed mutagenesis using wild-type (WT) NEAT1 promoter, MALAT1 enhancer or UCA1 enhancer vectors as templates. The primers used are described in Additional file 1: Table S4.

For luciferase activity assays, cells were seeded the day before transfection. The pGL4-Renilla construct was included as an internal control. After $16 \mathrm{~h}$ of co-transfection with empty vector or gene promoter/enhancer vector, and pGL3-Basic or pGL4-Renilla, the dual luciferase reporter assay kit (Promega, Madison, WI, USA) was used to determine gene promoter or enhancer activity. The luminescence was measured with a Turner BioSystems luminometer (Promega). The data are represented as the means of ratio of firefly luciferase to Renilla luciferase activity by triplicate experiments.

\section{RealTime-Glo viability assay}

Cell viability was assayed using RealTime-Glo assay (Promega). Briefly, cells were transfected for $24 \mathrm{~h}$ and then reseeded at $2 \times 10^{3}$ cells/well in 96-well plates. MT Cell Viability Substrate and NanoLuc Enzyme were diluted and added to each well. The luminescence was measured with a Turner BioSystems luminometer (Promega) at 24, 48 and $72 \mathrm{~h}$.

\section{Transwell migration and invasion assay}

The transwell insert with millipore membrane (pore size of $8 \mu \mathrm{m}$, Falcon, BD Franklin Lakes, NJ, USA) was used. For transwell migration assay, $2 \times 10^{5}$ A549 cells and $5 \times 10^{5}$ CL1-0 cells were seeded onto the upper chamber with $1 \mathrm{ml}$ serum-free medium. For transwell invasion assay, the transwell inserted membranes were pre-coated with Matri-gel $(2.5 \mathrm{mg} / \mathrm{ml}$, Sigma-Aldrich, St. Louis, MO, USA) 1 day before seeding cells.
Complete medium with 20\% FBS was supplemented into the lower chamber as chemoattractants. The cells were incubated for $16 \sim 24 \mathrm{~h}$ and then the cells attached on the reverse side of the membrane were then fixed and stained. Six random views were photographed and quantified under an upright microscope (Nikon E400, Yurakucho, Tokyo, Japan).

\section{Study population}

We recruited 124 lung cancer patients from National Cheng Kung University Hospital after obtaining appropriate institutional review board permission and informed consent from the patients. Surgically resected tumor tissue and corresponding normal tissue samples were collected. Total RNA of patient samples were prepared using Trizol reagent (Invitrogen) and reverse transcribed into cDNA as described above. qRT-PCR was conducted to measure the expressions of Oct4, NEAT1 and MALAT1 using the StepOnePlus $^{\text {тм }}$ Real-Time PCR System (Applied Biosystems). The expression of the target genes was normalized based on the levels of internal control gene, GAPDH. The primers used for qRT-PCR analyses are described in Additional file 1: Table S3.

\section{Statistical analysis}

Pearson $\chi^{2}$ test was used to compare the correlation of Oct4 and lncRNAs expression and clinicopathological parameters in lung cancer patients. Overall survival curves were calculated according to the Kaplan-Meier method, and comparison was performed using the log-rank test. Two-way ANOVA and two-tailed Student's $t$-test was used in cell and animal studies. Data represent mean \pm SEM. $P<0.05$ was considered to be statistically significant.

\section{Results}

Unbiased ChIP-seq and ChIP-PCR/qRT-PCR analyses reveal novel IncRNAs controlled by Oct4 transcriptional regulation in lung cancer

We previously performed ChIP-seq in A549 lung cancer cell line stably-overexpressing Oct4 to identify the Oct4 genome-wide DNA binding regions [8]. Notably, genomic loci of some lncRNAs were bound by Oct4. We further identified the potential Oct4-regulated lncRNAs through the following bioinformatic and functional analyses. Firstly, we integrated our in-house ChIP-seq dataset with functional lncRNA database [20] and lncRNA $\mathrm{db}$ [21] with literature annotation to search for lncRNAs with oncogenic potential. Secondly, candidate lncRNAs were further selected following the criteria that the Oct4 binding sites located within $100 \mathrm{~kb}$ from lncRNAs genomic loci. We then performed ChIP-PCR and qRT-PCR in A549 and CL1-0 cells overexpressing Oct4 to validate 
the selected lncRNAs for Oct4 protein binding and transcriptional regulation, seven oncogenic lncRNAs were validated (Fig. 1a). A549 and CL1-0 lung cancer cells were confirmed for the Oct4-induced oncogenic effects in vitro and in vivo (Additional file 1: Figure S1). Visualization of Oct4 ChIP-seq targeting revealed significant enrichments of Oct4 binding on three lncRNAs, including the promoter region of NEAT1 and enhancer regions of MALAT1 and $U C A 1$ (Fig. 1b).

To validate ChIP-seq data, we performed ChIP-PCR and qRT-PCR to elucidate whether Oct4 transcriptionally regulates the eight selected lncRNAs, including seven oncogenic lncRNAs BACE1AS, IGF2AS, MALAT1, NEAT1, SRA, TUG1 and UCA1, and tumor suppressor

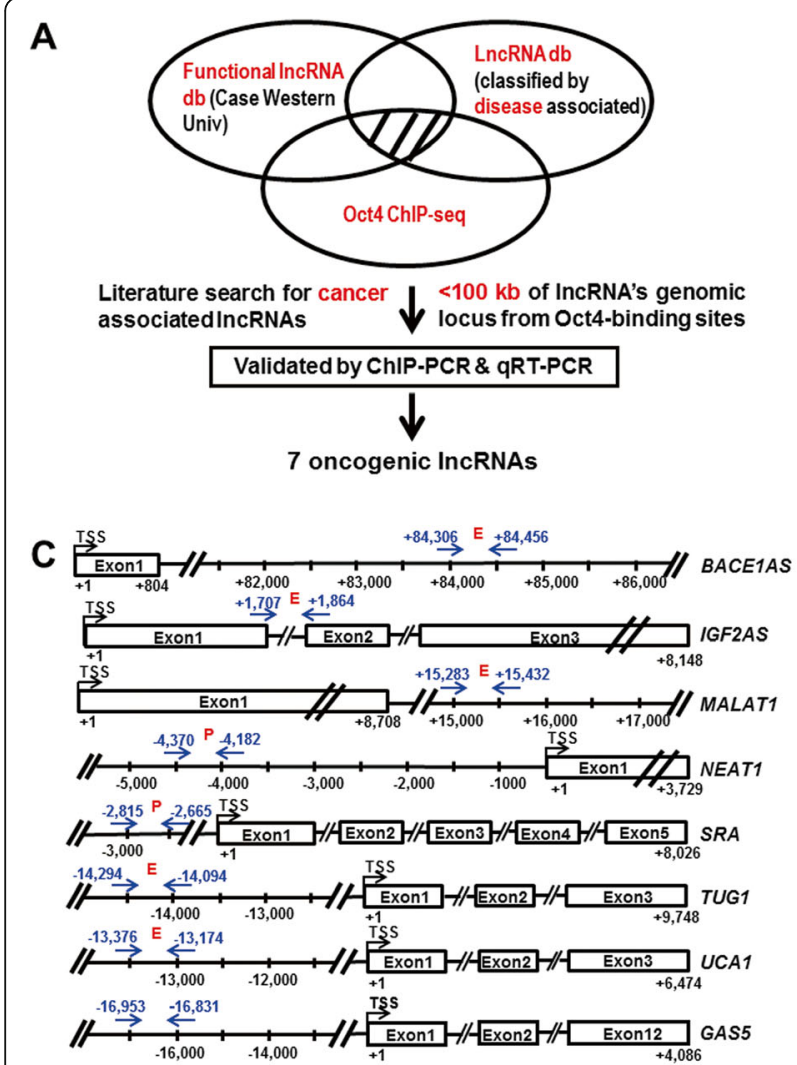

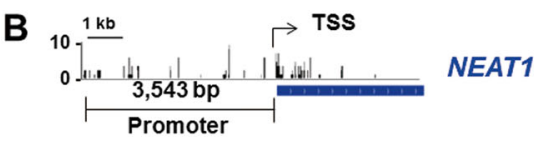
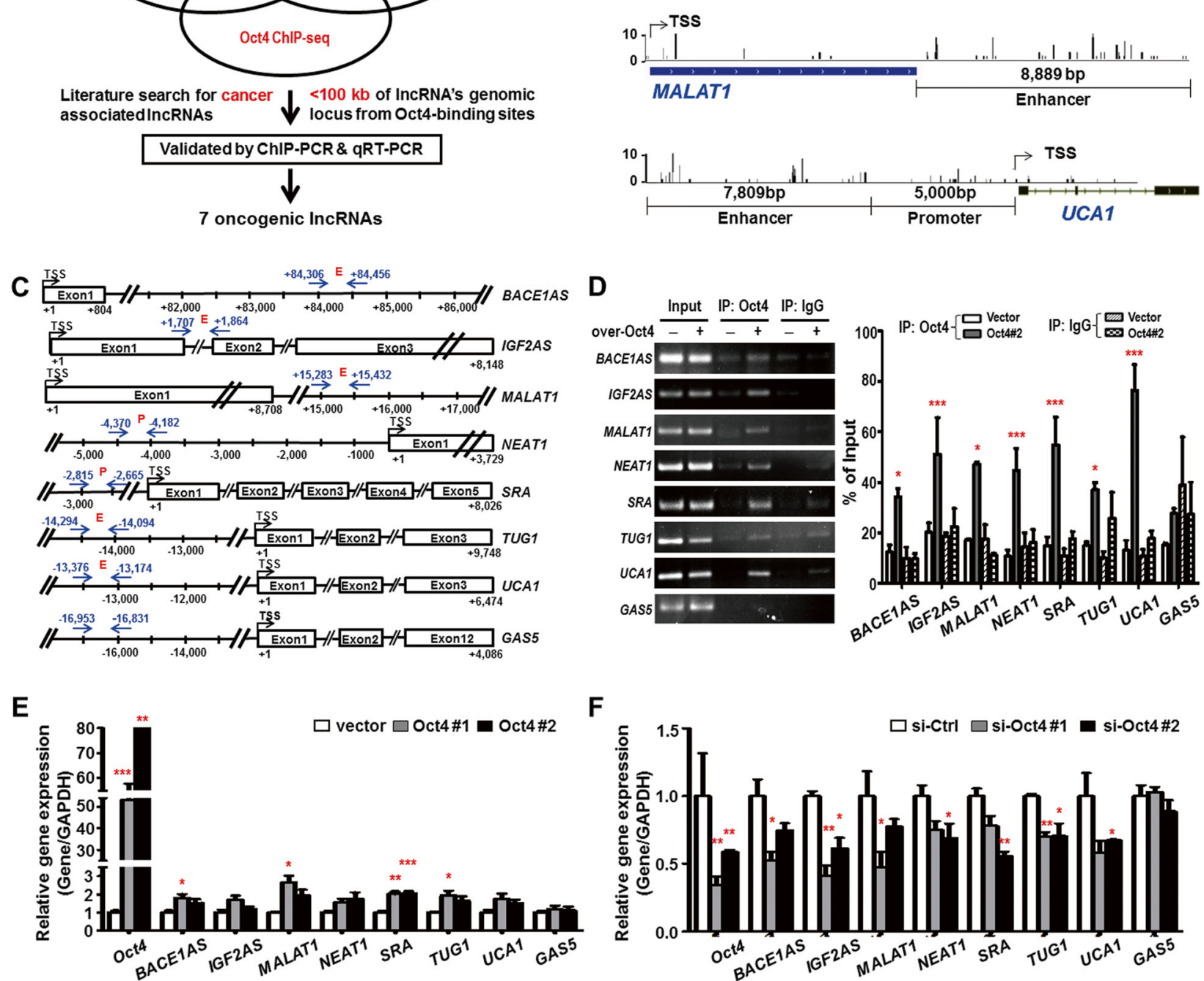

Fig. 1 Oncogenic IncRNAs were revealed as Oct4 transcriptional downstream targets in lung cancer using ChIP-seq, ChIP-PCR and QRT-PCR assays. a Flowchart of strategies used to identify Oct4 targeted downstream IncRNAs. $\mathbf{b}$ The Oct4 binding peaks at promoter and enhancer regions of three representative IncRNAs NEAT1, MALAT1 and UCA1. c Schematic diagram depicting the ChIP-PCR primers for amplification of the regions including Oct4 binding sites around the eight IncRNAs genomic locus (indicated as blue arrows). TSS: transcription start site as indicated by (+1). Oct4 binding regions were classified as enhancer (E) or promoter (P). $\mathbf{d}$ ChIP-PCR analysis of Oct4 occupancy at the binding sites of the eight IncRNAs genomic loci. GAS5 serves as a negative control IncRNA. Results are normalized to input by semi-quantitative analysis. IgG serves as an experimental negative control. Data represent mean \pm SEM. P-values were determined by two-way ANOVA. e, $\mathbf{f}$ qRT-PCR analysis of eight IncRNAs expressions in A549 cells stably overexpressing Oct4 (Oct4\#1, Oct4\#2) (e) or Oct4-silenced A549 cells (si-Oct4\#1, si-Oct4\#2) (f). GAS5 serves as a negative control IncRNA. Target IncRNA expression levels were normalized to GAPDH expression levels. Data represent mean \pm SEM. $P$-values were determined by two-tailed Student t-test. ${ }^{*} P<0.05 ;{ }^{* *} P<0.01 ;{ }^{* * *} P<0.001$ 
lncRNA GAS5. The GAS5 was selected as a negative control lncRNA because it contained no ChIP-seq binding signal of Oct4. We used ALGGEN PROMO and TFSEARCH softwares to identify the putative Oct4 consensus binding elements (5'ATGCAAAT3') of
ChIP-seq regions. Oct4 binding sites within $5 \mathrm{~kb}$ upstream of transcription start site (TSS) were defined as promoter region, whereas Oct4 binding regions located at more than $5 \mathrm{~kb}$ of TSS or within the gene body were defined as enhancer region according to

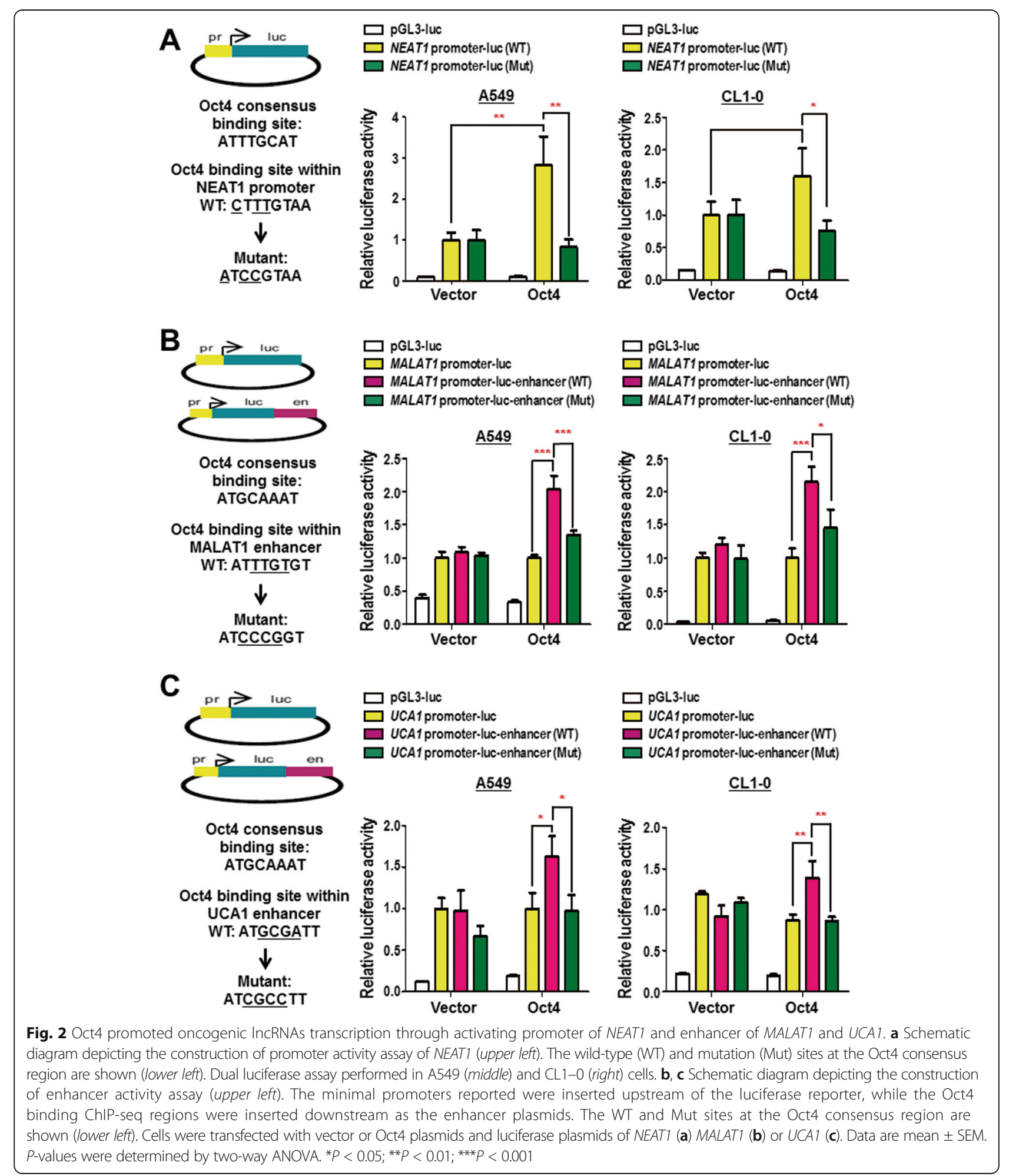


previous studies $[22,23]$. Therefore, we classified the Oct4 binding sites to promoter $(\mathrm{P})$ and enhancer $(\mathrm{E})$ in relation to TSS of the seven lncRNAs (Fig. 1c). The ChIP-PCR results showed that Oct4 indeed targeted the predicted promoter or enhancer regions of the oncogenic lncRNAs but not the negative control lncRNA GAS5 (Fig. 1d).

To further confirm the transcription activation of Oct4 on downstream lncRNAs, qRT-PCR analysis was conducted in both A549 and CL1-0 cells stably expressing Oct4. Results showed that overexpression of Oct4 in A549 increased the expression of the seven oncogenic
IncRNAs but not that of the IncRNA GAS5 (Fig. 1e). In contrast, knockdown of Oct4 in A549 cells decreased expression of the oncogenic lncRNAs but not that of GAS5 (Fig. 1f). The results in CL1-0 cells showed a similar trend but to a lesser extent compared to that in A549 cells (Additional file 1: Figure S2). An increased expression of NEAT1 and UCA1 lncRNAs upon Oct4 overexpression was observed in the normal bronchial epithelial cell line BEAS-2B (Additional file 1: Figure S3). These results suggested that Oct 4 may transcriptionally upregulate the expression of these oncogenic lncRNAs identified.

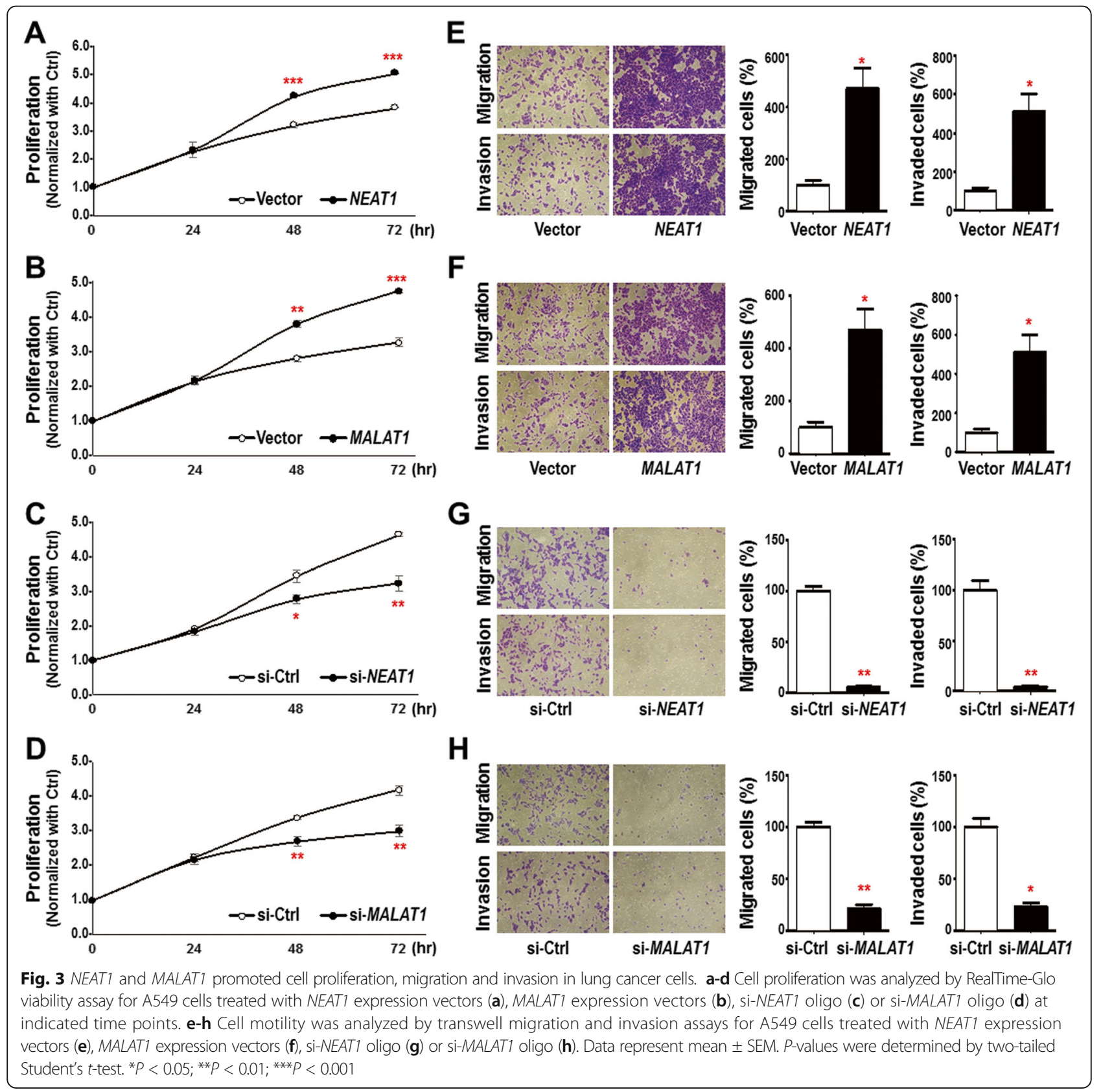


Oct4 transcriptionally activates NEAT1 via promoter and activates MALAT1 and UCA1 via enhancer binding

To investigate the mechanism underlying Oct4-mediated lncRNAs upregulation, we selected NEAT1, MALAT1 and UCA1 lncRNAs for further transcriptional analyses. Oct4 was validated to bind at NEAT1 promoter region while to $M A L A T 1$ and $U C A 1$ at their enhancer regions (Fig. 1c and d). NEAT1 promoter derived from the Oct4 binding ChIP-seq region was inserted upstream of the luciferase reporter gene (Fig. 2a, left). A549 and CL1-0 cells transiently overexpressing Oct4 significantly induced NEAT1 promoter activity (Fig. 2a, yellow bars). However, Oct4 did not affect the activity of NEAT1 promoter with Oct4 binding element mutated in both lung cancer cells (Fig. 2a, green bars). As for the enhancer construction, reported minimal promoter activity fragment of MALAT1 or UCA1 [24, 25] and the enhancer fragment with Oct4 binding ChIP-seq region of MALAT1 or UCA1 was constructed to the upstream and downstream of luciferase reporter, respectively (Fig. 2b and c, left). A549 and CL1-0 cells transiently overexpressing Oct4 potentiated MALAT1 and $U C A 1$ enhancer activities compared with activities with promoter alone (Fig. 2b and c, bars pink vs. yellow). However, the Oct4-induced enhancer activity was attenuated when the Oct4 binding element within MALAT1 or UCA1 enhancer was mutated in A549 and CL1-0 cells (Fig. 2b and c, bars green vs. pink). Together, we have demonstrated that Oct4 directly regulated NEAT1, MALAT1 and UCA1 lncRNAs transcription through targeting their promoter or enhancer regions.

\section{NEAT1 and MALAT1 are downstream effectors of Oct4-induced lung cancer proliferation, migration and invasion}

Since oncogenic lncRNAs, NEAT1 and MALAT1, were positively regulated by Oct4 at transcriptional level, we further characterized the oncogenic roles of NEAT1 and MALAT1 lncRNAs in lung cancer cells. Transiently overexpressing NEAT1 or MALAT1 in A549 cells indeed promoted cell proliferation (Fig. $3 \mathrm{a}$ and b). In contrast, knockdown of NEAT1 or MALAT1 expression suppressed cell proliferation in A549 cells (Fig. 3c and d). In addition, transwell migration and invasion assays confirmed that NEAT1 or MALAT1 overexpression in A549 cells enhanced cell migration and invasion (Fig. 3e and f), while knockdown of NEAT1 or MALAT1 suppressed cell migration and invasion (Fig. $3 \mathrm{~g}$ and h). These results indicated that NEAT1 and MALAT1 indeed exerted oncogenic

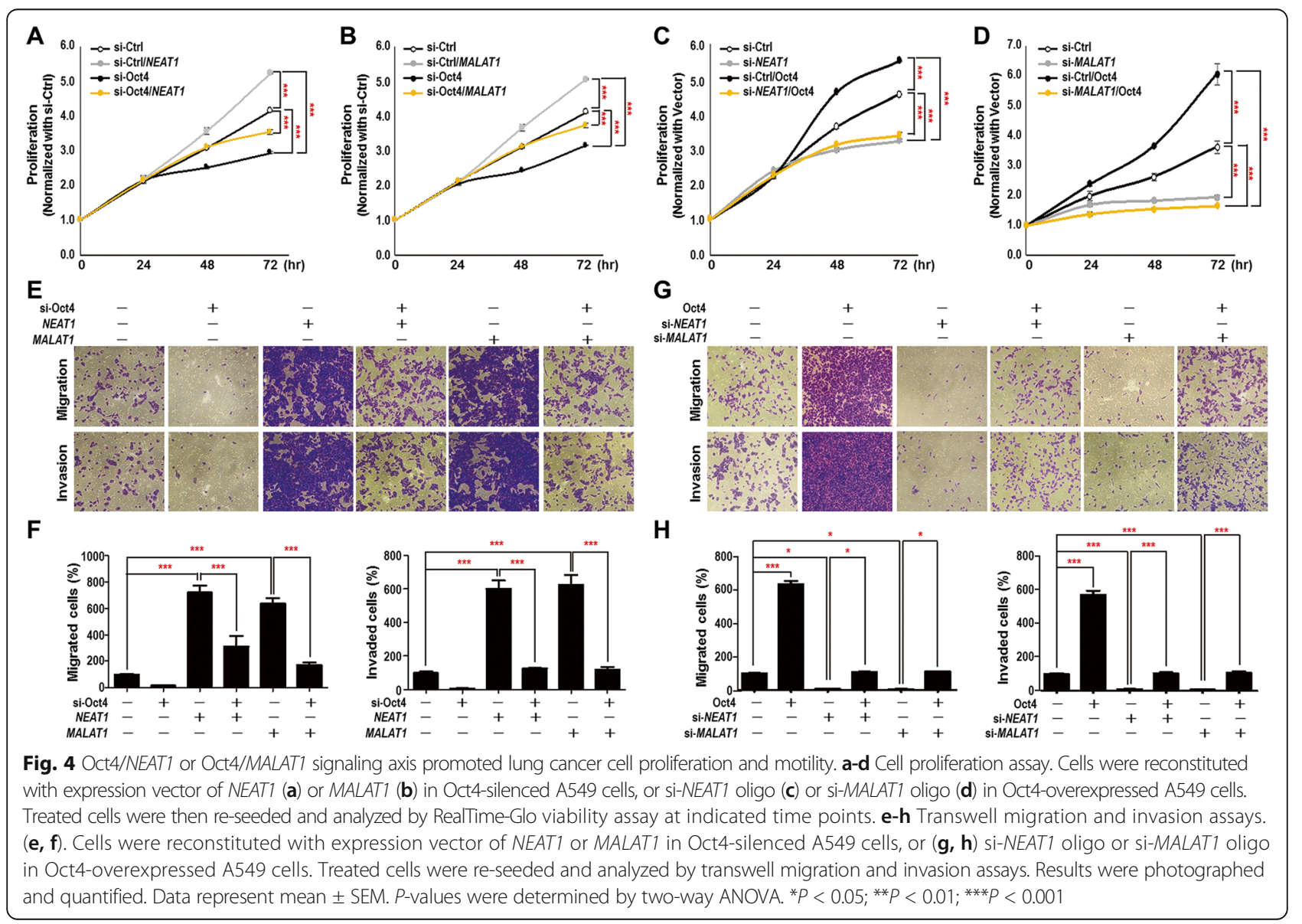


effects in lung cancer cells. The expression levels of NEAT1 and MALAT1 manipulation in functional experiments were confirmed using qRT-PCR analysis and are shown in Additional file 1: Figure S4.

To further elucidate whether NEAT1 and MALAT1 contributed to Oct4-mediated oncogenic effects, we conducted reconstitution experiments by transfecting A549 cells with Oct4 expression plasmid alone or together with si-NEAT or si-MALAT1 oligo. On the other hand, A549 cells were transfected with si-Oct4 oligo alone or together with NEAT1 or MALAT1 expression vectors. The expression level of Oct4, NEAT1 and MALAT1 manipulation in reconstitution experiments were confirmed using Western blot and qRT-PCR analyses and results are shown in Additional file 1: Figure S5. The transfected cells were also subjected to proliferation assay, transwell migration and invasion assays. Our results showed that reconstituted expression of NEAT1 or MALAT1 recovered proliferation (Fig. 4a and $\mathrm{b}$ ), migration and invasion (Fig. 4e and $\mathrm{f}$ ) abilities, which was downregulated in Oct4-knockdown A549 cells. In contrast, knockdown of NEAT1 or MALAT1 abolished Oct4-promoted A549 cells proliferation (Fig. 4c and d), migration and invasion (Fig. $4 \mathrm{~g}$ and $\mathrm{h}$ ) abilities. The results suggested that Oct4/NEAT1 and Oct4/MALAT1 transcriptional axes promote oncogenic effects in lung cancer.

\section{Clinical significance of coinciding high expressions of Oct4, NEAT1 and MALAT1 in lung cancer patients}

To further validate Oct4/NEAT1 and Oct4/MALAT1 transcriptional axes in lung cancer patients, we examined RNA expression level of Oct4, NEAT1 and MALAT1 using qRT-PCR analysis of samples from 124 lung cancer patients. The overexpression rate for Oct4, NEAT1 and MALAT1 RNA were $85.5 \%, 90.3 \%$ and $88.7 \%$, indicating the oncogenic roles of Oct4, NEAT1 and MALAT1 in lung cancer patients (Table 1). Of note, significant positive correlations were found between Oct4 mRNA and NEAT1 or between Oct4 mRNA and MALAT1 lncRNA expression $(P<0.001$ for Oct4 and NEAT1; $P<0.001$ for Oct4 and MALAT1) (Table 1).

To determine whether high expression of Oct4, NEAT1 and MALAT1 contributes to poor outcome in lung cancer patients, Kaplan-Meier analysis was performed and data showed that high expression of Oct4

Table 1 Alteration of Oct4, NEAT1 and MALAT1 expression levels in relation to clinicopathological parameters in 124 lung cancer patients

\begin{tabular}{|c|c|c|c|c|c|c|c|c|c|c|c|c|c|c|c|c|}
\hline \multirow[t]{3}{*}{ Characteristics } & \multirow{3}{*}{$\begin{array}{l}\text { Total } \\
\mathrm{n}\end{array}$} & \multicolumn{4}{|c|}{ Oct4 } & \multirow[b]{3}{*}{$p^{a}$} & \multicolumn{4}{|c|}{ NEAT1 } & \multirow[b]{3}{*}{$P^{a}$} & \multicolumn{4}{|c|}{ MALAT1 } & \multirow[b]{3}{*}{$p^{\mathrm{a}}$} \\
\hline & & \multicolumn{2}{|c|}{$\overline{\text { Normal }}$} & \multicolumn{2}{|c|}{ Overexpression } & & \multicolumn{2}{|c|}{ Normal } & \multicolumn{2}{|c|}{ Overexpression } & & \multicolumn{2}{|c|}{$\overline{\text { Normal }}$} & \multicolumn{2}{|c|}{ Overexpression } & \\
\hline & & $n$ & (\%) & $\mathrm{n}$ & (\%) & & n & (\%) & n & (\%) & & n & (\%) & n & (\%) & \\
\hline Overall & 124 & 18 & $(14.5)$ & 106 & $(85.5)$ & & 12 & (9.7) & 112 & $(90.3)$ & & 14 & $(11.3)$ & 110 & $(88.7)$ & \\
\hline \multicolumn{17}{|l|}{ Tumor stage } \\
\hline $\mid+\|$ & 72 & 16 & $(22.2)$ & 56 & (77.8) & 0.005 & 11 & (15.3) & 61 & $(84.7)$ & 0.014 & 12 & (16.7) & 60 & (83.3) & 0.028 \\
\hline$I I I+I V$ & 51 & 2 & (3.9) & 49 & $(96.1)$ & & 1 & (2.0) & 50 & $(98.0)$ & & 2 & (3.9) & 49 & $(96.1)$ & \\
\hline \multicolumn{17}{|l|}{ T stage } \\
\hline $\mid+\|$ & 104 & 18 & $(17.3)$ & 86 & $(82.7)$ & 0.050 & 11 & (10.6) & 93 & $(89.4)$ & 0.473 & 13 & (12.5) & 91 & $(87.5)$ & 0.361 \\
\hline$I I I+I V$ & 19 & 0 & $(0.0)$ & 19 & $(100.0)$ & & 1 & (5.3) & 18 & $(94.7)$ & & 1 & (5.3) & 18 & $(94.7)$ & \\
\hline \multicolumn{17}{|l|}{$N$ stage $^{b}$} \\
\hline 0 & 71 & 15 & $(21.1)$ & 56 & $(78.9)$ & 0.021 & 11 & (15.5) & 60 & $(84.5)$ & 0.014 & 12 & $(16.9)$ & 59 & $(83.1)$ & 0.029 \\
\hline $1+2$ & 50 & 3 & $(6.0)$ & 47 & $(94.0)$ & & 1 & $(2.0)$ & 49 & $(98.0)$ & & 2 & $(4.0)$ & 48 & $(96.0)$ & \\
\hline \multicolumn{17}{|l|}{ M stage $e^{c}$} \\
\hline 0 & 116 & 18 & $(15.5)$ & 98 & $(84.5)$ & 0.259 & 12 & (10.3) & 104 & $(89.7)$ & 0.370 & 14 & (12.1) & 102 & $(87.9)$ & 0.329 \\
\hline 1 & 7 & 0 & $(0.0)$ & 7 & $(100.0)$ & & 0 & $(0.0)$ & 7 & $(100.0)$ & & 0 & $(0.0)$ & 7 & $(100.0)$ & \\
\hline \multicolumn{17}{|l|}{ NEAT1 } \\
\hline Normal & 12 & 10 & $(83.3)$ & 2 & $(16.7)$ & $<0.001$ & - & - & - & - & - & - & - & - & - & \\
\hline Overexpression & 112 & 8 & $(7.1)$ & 104 & $(92.9)$ & & - & - & - & - & & - & - & - & - & \\
\hline \multicolumn{17}{|l|}{ MALAT1 } \\
\hline Normal & 14 & 10 & $(71.4)$ & 4 & (28.6) & $<0.001$ & 11 & (78.6) & 3 & $(21.4)$ & $<0.001$ & - & - & - & - & \\
\hline Overexpression & 110 & 8 & (7.3) & 102 & $(92.7)$ & & 1 & (0.9) & 109 & (99.1) & & - & - & - & - & \\
\hline
\end{tabular}

${ }^{a}$ The data were analyzed by Pearson $\times 2$ test. Bold values indicate statistical significance $(P<0.05)$

${ }^{\mathrm{b}} \mathrm{N}$ Stage: lymph node metastasis

${ }^{\mathrm{c}} \mathrm{M}$ Stage: distant metastasis 
mRNA $(P=0.01)$, NEAT1 lncRNA $(P=0.006)$ or MALAT1 lncRNA $(P=0.014)$ in lung cancer patients was associated with poor overall survival (Fig. 5a-c). Moreover, patients with coinciding high expression of Oct4, NEAT1 and MALAT1 had poorer prognosis compared with other patients $(P=0.002)$ (Fig. $5 \mathrm{~d})$. Univariate Cox regression analysis confirmed that patients with co-overexpressed Oct4, NEAT1 and MALAT1 had poor outcome $(P=0.016$, hazard ratio $=2.78,95 \%$ confidence interval $=1.21-6.42$; Table 2, left panel). After adjusting for late stage and lymph node metastasis using multivariate Cox regression analysis, co-overexpression of Oct4, NEAT1 and MALAT1 in lung cancer patients showed a relative risk of death of $2.42(P=0.039$; Table 2 , right panel). These clinical studies clearly indicated that Oct4 positively correlates with NEAT1 and MALAT1 expression in lung cancer and that Oct4/NEAT1/MALAT1 co-overexpression is an independent factor for prediction of poor outcome in lung cancer patients.

\section{Discussion}

In the present study, we have revealed that Oct4 binds to the genomic loci of IncRNAs through ChIP-seq and bioinformatic analysis (Fig. 1). We then validated that Oct4 bound on the promoter or enhancer regions of lncRNAs (Fig. 1). Dual luciferase activity assay further confirmed that Oct4 potentiated promoter activity of NEAT1 and enhancer activities of MALAT1 and UCA1
lncRNAs (Fig. 2). Moreover, NEAT1 and MALAT1 acted as downstream effectors of Oct4 to promote proliferation, migration and invasion abilities of A549 lung cancer cells (Figs. 3 and 4). Of note, positive correlations between Oct4 mRNA and NEAT1/MALAT1 lncRNAs were evident in lung cancer patient specimens (Fig. 5). Our study provides new evidence that Oct 4 transcriptionally regulates lncRNAs expression by targeting their promoter or enhancer regions. NEAT1 and MALAT1 function as Oct4 downstream mediators to promote lung cancer proliferation, migration and invasion (Fig. 5e).

Recently, the roles of NEAT1 in cancer have been uncovered. Studies have reported that NEAT1 is upregulated in prostate cancer, colorectal cancer and lung cancer, and thus associated with poor prognosis in these cancer patients [16, 26-28]. Rubin and associates demonstrated that estrogen receptor transcriptionally activates NEAT1 expression to promote prostate tumorigenesis under the treatment of oestrogen [16]. NEAT1 has also been shown to modulate prostate cancerspecific gene expression through chromatin modifications and thus contributes to cancer progression [16]. However, the upstream mechanisms of NEAT1 overexpression in cancers await to be uncovered. It is until recently that HIF- $2 \alpha$ is demonstrated to transactivate NEAT1 transcription under hypoxia, which promotes the formation of paraspeckles, accelerates tumor proliferation and cancer cell survival leading to poor

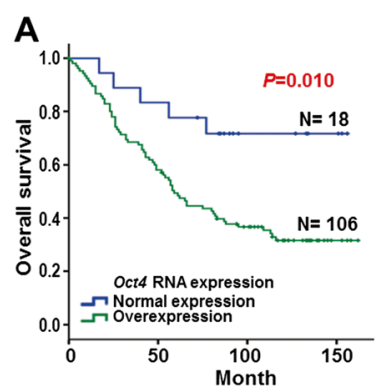

D

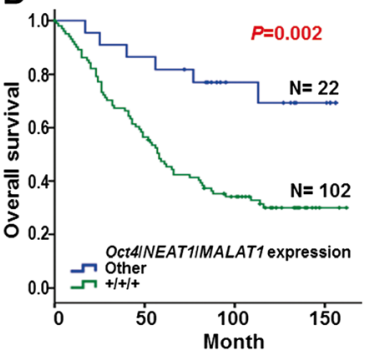

B

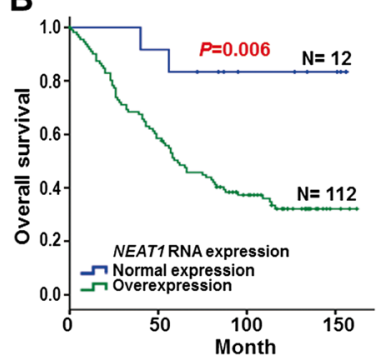

E

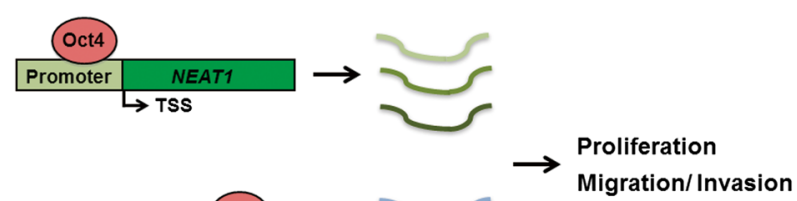

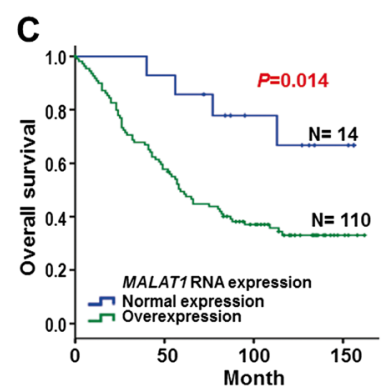

Migration/Invasion

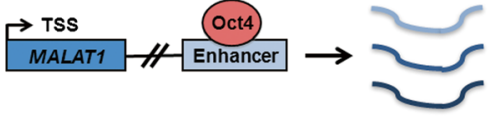

Fig. 5 Clinical significances of Oct4/NEAT1/MALAT1 signaling axis in 124 lung cancer patients. qRT-PCR analyses of Oct4, NEAT1 and MALAT1 RNA expression were performed in 124 lung cancer specimens. a-d Overall survival analyzed by Kaplan-Meier method indicated that patients with high RNA expression of Oct4 (a), NEAT1 (b), MALAT1 (c) or coinciding high expression of Oct4, NEAT1 and MALAT1 (d) had poor survival. $P$-values were determined using log-rank test. e Schematic diagram depicting Oct4 activates NEAT1 transcription via promoter binding and MALAT1 transcription via enhancer binding. NEAT1 and MALAT1 IncRNAs act as downstream effectors of Oct4 to promote lung cancer proliferation, migration and invasion, and thereby lead to lung cancer progression 
Table 2 Cox regression analysis of risk factors for cancer-related death in lung cancer patients

\begin{tabular}{|c|c|c|c|c|}
\hline \multirow[t]{2}{*}{ Characteristics } & \multicolumn{2}{|c|}{$\underline{\text { Univariate analysis }}$} & \multicolumn{2}{|c|}{ Multivariate analysis } \\
\hline & $\mathrm{HR}^{\mathrm{a}}\left(95 \% \mathrm{Cl}^{\mathrm{b}}\right)$ & $P$-value ${ }^{c}$ & $\mathrm{HR}^{\mathrm{a}}\left(95 \% \mathrm{Cl}^{\mathrm{b}}\right)$ & $P$-value ${ }^{c}$ \\
\hline \multicolumn{5}{|c|}{ Oct4/NEAT1/MALAT1 expression } \\
\hline Other & 1.00 & & 1.00 & \\
\hline$+/+/+$ & $2.78(1.21-6.42)$ & 0.016 & $2.42(1.05-5.62)$ & 0.039 \\
\hline \multicolumn{5}{|l|}{ Age } \\
\hline$<65$ year-old & 1.00 & & $-e$ & \\
\hline$\geq 65$ year-old & $1.03(0.64-1.67)$ & 0.896 & $-e$ & $-e$ \\
\hline \multicolumn{5}{|l|}{ Gender } \\
\hline Female & 1.00 & & $-{ }^{e}$ & \\
\hline Male & $1.50(0.86-2.61)$ & 0.150 & $-e^{e}$ & $-e^{e}$ \\
\hline \multicolumn{5}{|l|}{ Smoking habit } \\
\hline Non-smoker & 1.00 & & - e & \\
\hline Smoker & $0.96(0.54-1.72)$ & 0.891 & $-{ }^{e}$ & $-e^{e}$ \\
\hline \multicolumn{5}{|l|}{ Tumor type $^{d}$} \\
\hline SCC & 1.00 & & $-e$ & \\
\hline ADC & $1.32(0.74-2.37)$ & 0.343 & $-e^{e}$ & $-e$ \\
\hline \multicolumn{5}{|l|}{ Stage } \\
\hline Stage I-II & 1.00 & & 1.00 & \\
\hline Stage III-IV & $1.86(1.18-2.95)$ & 0.008 & $1.13(0.65-1.97)$ & 0.672 \\
\hline \multicolumn{5}{|l|}{ T stage } \\
\hline $\mathrm{T} 1-2$ & 1.00 & & $-{ }^{e}$ & \\
\hline T3-4 & $1.42(0.78-2.59)$ & 0.249 & $-{ }^{e}$ & $-e^{e}$ \\
\hline \multicolumn{5}{|l|}{$\mathrm{N}$ stage } \\
\hline No & 1.00 & & 1.00 & \\
\hline$\geq \mathrm{N} 1$ & $2.36(1.48-3.78)$ & $<0.001$ & $1.93(1.10-3.38)$ & 0.022 \\
\hline \multicolumn{5}{|l|}{ M stage } \\
\hline MO & 1.00 & & $-e$ & \\
\hline$\geq M 1$ & $1.98(0.86-4.57)$ & 0.110 & $-{ }^{e}$ & $-^{e}$ \\
\hline
\end{tabular}

${ }^{a} H R$ hazard ratio

${ }^{\mathrm{b}} \mathrm{Cl}$ confidence interval

${ }^{c}$ Bold values indicate statistical significance $(P<0.05)$

${ }^{\mathrm{d} S C C}$ squamous cell carcinoma, $A D C$ adenocarcinoma

${ }^{\mathrm{e}}$ The variables without significant HR in the univariate analysis were not included in the multivariate analysis

prognosis in breast cancer patient [29]. In our study, we have shown that a well-known stemness transcription factor Oct4 transcriptionally upregulates NEAT1 expression through binding to Oct4 consensus binding element on promoter region (Figs. 1 and 2a), and therefore promoting lung cancer proliferation and motility (Figs. 3 and 4). Notably, NEAT1 is found overexpressed in BRCA1-deficient breast cancer and promotes self-renewal abilities in breast cancer cells through epigenetically suppressing miR-1295p, which targets to Wnt4 [30]. The last-mentioned study together with our results revealed a potential role of NEAT1 in maintaining stemness properties and suggested that Oct4-mediated NEAT1 upregulation may play critical roles in embryonic or cancer stemness maintenance.

$M A L A T 1$ was first identified as a prognosis marker in early-stage metastasizing lung cancer [31]. MALAT1 knockdown in lung cancer cells decreases cell migration abilities [32]. In addition, MALAT1 suppresses expression of anti-metastasis genes such as MIA2 (melanoma inhibitory activity 2) and $R O B O 1$ (roundabout 1), while induces pro-metastasis genes including LPHN2 (latrophilin 2) and $A B C A 1$ (ATP-binding cassette, sub-family A, member 1) to accelerate metastasis [33]. However, $M A L A T 1$-promoting lung cancer cell proliferation in different studies are contradictory. For example, MALAT1 has no effect on cell proliferation in vitro and slightly promotes tumor growth in vivo [33]. In contrast, knockdown of MALAT1 in A549 lung cancer cells decreased proliferation [34], which is consistent with our results that MALAT1 plays a role in lung cancer cell proliferation (Fig. $3 \mathrm{~b}$ and $\mathrm{d}$ ) and this provides new insight into the role of MALAT1 in various cancer types. MALAT1 has been demonstrated to promote lung, bladder, colorectal, liver, oral and prostate cancer cells proliferation and migration [32, 33, 35-40]. However, the upstream regulatory mechanisms of MALAT1 expression remain unclear, especially at the transcription level. Recently, Sp1 is found to transcriptionally activate MALAT1 expression through targeting the promoter region and the Sp1-MALAT1 axis may play a critical role in cancers [34]. In addition, Wnt signaling pathway acts upstream of MALAT1 transcription, which is mediated by TCF4 binding on MALAT1 promoter in endometrioid endometrial cancer [41]. Importantly, our results provide the first evidence of Oct4-mediated MALAT1 upregulation through enhancer regions.

\section{Conclusions}

In conclusion, our genome-wide ChIP-seq analysis reveals a novel role of Oct4 transcription regulation on lncRNAs in lung cancer. We demonstrate for the first time that Oct4 promotes transcription of NEAT1, $M A L A T 1$ and $U C A 1$ through targeting their promoter or enhancer regions. Moreover, NEAT1 and MALAT1 function as Oct4 downstream mediators to promote lung cancer proliferation, migration and invasion. Clinical studies confirm that patients with Oct4/NEAT1/MALAT1 high expression had poor outcome. Collectively, our study provides a novel insight into Oc4-IncRNAs as critical axes in lung tumorigenesis.

\section{Additional file}

Additional file 1: Table S1. Supplementary methods. Anchorageindependent growth assay, Tumor-sphere formation assay, Tumor formation assay, Western blot analysis. Table S1. The plasmids and their characteristics 
used in the current study. Table S2. The ChIP-PCR primers used in the current study. Table S3. The CDNA primers used in the current study. Table S4. The construction primers of promoters and enhancers used in the current study. Figure S1. Oct4 promoted lung cancer tumorigenesis in vitro and in vivo. A Anchorage-independent assays in empty vector stably-transfected cell line (vector) and two biological replicates of Oct4 stably-overexpressed A549 and CL1-0 cell lines (Oct4\#1, Oct4\#2). Results were photographed (left) and quantified (right). B Transwell migration and invasion assay analysis of stably-transfected cell lines in A549 and CL1-0 cells. Results were photographed (left) and quantified (right). P-values were determined by two-tailed Student's t-test. ${ }^{*} P<0.05 ;{ }^{*} P<0.01 ;{ }^{* *} P<0.001$. C In vitro tumor sphere formation assay of A549 lung cancer cells stably expressing Oct4\#1 or vector photographed (top) and quantified (middle). In vivo tumor formation assay using limited cell number $(100,1000$, and 5000 cells) of vector and Oct4\#1 cells. Tumor incidence of mice was analyzed at 8 weeks after implantation. D The immunoblots (upper) and qRT-PCR (lower) confirmed Oct4 expression in A549 and CL1-0 stable clones. Figure S2. Expression of IncRNAs in CL1-0 lung cancer cells manipulated for Oct4. A, B qRT-PCR analysis of eight IncRNAs expression in CL1-0 cells stably overexpressing Oct4 (Oct4\#1, Oct4\#2) (A) or Oct4-silenced CL1-0 cells (si-Oct4\#1, si-Oct4\#2) (B). Target IncRNA expression levels were normalized to GAPDH expression levels. Data represent mean \pm SEM. $P$-values were determined by two-tailed Student's $t$-test. ${ }^{*} P<0.05$; ${ }^{*} P<0.01 ;{ }^{* * *} P<0.001$. Figure $\mathbf{S 3}$. Oct4 positively regulated NEAT1 and UCA1 IncRNAs transcription in normal bronchial epithelial BEAS-2B cells. qRT-PCR analysis of selected IncRNAs expressions in BEAS-2B cells overexpressing Oct4. Target IncRNA expression levels were normalized to GAPDH expression levels. Data represent mean \pm SEM. $P$-values were determined by two-tailed Student's $t$-test. ${ }^{*} P<0.05 ;{ }^{*} P<0.01$; ${ }^{* * *} P<0.001$. Figure S4. RNA expression level of the manipulated NEAT1 and MALAT1 in A549 lung cancer cells. A549 cells transfected with NEAT1 expression vector (A) or MALAT1 expression vector (B), si-NEAT1 oligo (si-NEAT1) (C) or si-MALAT1 oligo (si-MALAT1) (D) were harvested and subjected to GRT-PCR assays for NEAT1 and MALAT1 RNA expression. Data are mean \pm SEM. $P$-values were determined by two-tailed Student's $t$-test. ${ }^{*} P<0.05 ;{ }^{* *} P<0.001$. Figure S5. RNA and protein expression level of the manipulated Oct4, NEAT1 and MALAT1 in A549 lung cancer cells. A549 cells were transfected with expression vectors of NEAT1 (A) or MALAT1 (B) alone or together with si-Oct4 oligo (si-Oct4). A549 cells were transfected with si-NEAT1 oligo (si-NEAT1) (C) or si-MALAT1 oligo (si-MALAT1) (D) alone or together with Oct4 expression vector. Cell lysates were subjected to qRT-PCR assays for Oct4, NEAT1 and MALAT1 RNA expression or Western blot analysis for Oct4 protein expression (inset). GAPDH serves as an internal control. Data are mean \pm SEM. $P$-values were determined by two-way ANOVA. ${ }^{* * P}<0.01 ;{ }^{* * *} P<0.001$. (PDF $\left.911 \mathrm{~kb}\right)$

\section{Abbreviations}

ChIP: Chromatin-immunoprecipitation; IncRNA: Long non-coding RNA; MALAT1: Metastasis-associated lung adenocarcinoma transcript 1: NEAT1: Nuclear paraspeckle assembly transcript 1; POU5F1: POU domain, class 5, transcription factor 1; UCA1: Urothelial carcinoma-associated 1

\section{Acknowledgements}

We are grateful to Professors Ying Jin, Tetsuro Hirose and Kannanganattu V. Prasanth for generous help with the Oct4, NEAT1 and MALAT1 expression vectors. We thank the Human Biobank, Research Center of Clinical Medicine, National Cheng Kung University Hospital for providing the lung cancer specimens.

\section{Funding}

This work was supported by the Taiwan Ministry of Science and Technology Grant (MOST103-2320-B-006-045-MY3), the Taiwan Ministry of Health and Welfare Grant (MOHW103-TD-B-111-06) and the Aim for the Top University Project Grant (D103-35A02).

\section{Availability of data and materials}

All data used during the current study available from the corresponding author on reasonable request.

\section{Authors' contributions}

JJ, YAT, YHL and CCL performed the experiments. JJ, YAT, YHL and CCL did the data analysis in this study. WWL provided clinical samples. JJ, YHL and YCW wrote the paper. All authors read and approved the manuscript. YCW obtained funding.

\section{Competing interests}

The authors declare that they have no competing interests.

\section{Consent for publication}

Not applicable.

\section{Ethics approval and consent to participate}

This study was approved by the Institutional Review Board of National Cheng Kung University Hospital (B-ER-102-451), and patient consent was obtained prior to the initiation of the study.

\section{Publisher's Note}

Springer Nature remains neutral with regard to jurisdictional claims in published maps and institutional affiliations.

\section{Author details}

'Department of Pharmacology, College of Medicine, National Cheng Kung University, Tainan 701, Taiwan. ${ }^{2}$ Institute of Basic Medical Sciences, College of Medicine, National Cheng Kung University, Tainan 701, Taiwan. ${ }^{3}$ Division of Thoracic Surgery, Department of Surgery, National Cheng Kung University Hospital, College of Medicine, National Cheng Kung University, Tainan 701, Taiwan.

Received: 16 December 2016 Accepted: 4 June 2017 Published online: 14 June 2017

\section{References}

1. Wang Z, Oron E, Nelson B, Razis S, Ivanova N. Distinct lineage specification roles for NANOG, OCT4, and SOX2 in human embryonic stem cells. Cell Stem Cell. 2012;10:440-54

2. Chang CC, Shieh GS, Wu P, Lin CC, Shiau AL, Wu CL. Oct-3/4 expression reflects tumor progression and regulates motility of bladder cancer cells. Cancer Res. 2008;68:6281-91.

3. Ben-Porath I, Thomson MW, Carey VJ, Ge R, Bell GW, Regev A, et al. An embryonic stem cell-like gene expression signature in poorly differentiated aggressive human tumors. Nat Genet. 2008:40:499-507.

4. Kim BW, Cho H, Choi CH, Ylaya K, Chung JY, Kim JH, et al. Clinical significance of OCT4 and SOX2 protein expression in cervical cancer. BMC Cancer. 2015;15:1015.

5. Chiou SH, Yu CC, Huang CY, Lin SC, Liu CJ, Tsai TH, et al. Positive correlations of Oct-4 and Nanog in oral cancer stem-like cells and highgrade oral squamous cell carcinoma. Clin Cancer Res. 2008;14:4085-95.

6. Chai S, Ng KY, Tong M, Lau EY, Lee TK, Chan KW, et al. Octamer 4/ microRNA-1246 signaling axis drives Wnt/B-catenin activation in liver cancer stem cells. Hepatology. 2016;64:2062-76.

7. Karoubi G, Cortes-Dericks L, Gugger M, Galetta D, Spaggiari L, Schmid RA. Atypical expression and distribution of embryonic stem cell marker, OCT4 in human lung adenocarcinoma. J Surg Oncol. 2010;102:689-98.

8. Tang YA, Chen CH, Sun HS, Cheng CP, Tseng VS, Hsu HS, et al. Global Oct4 target gene analysis reveals novel downstream PTEN and TNC genes required for drug-resistance and metastasis in lung cancer. Nucleic Acids Res. 2015;43:1593-608.

9. $\mathrm{Ng} \mathrm{HH}$, Surani MA. The transcriptional and signalling networks of pluripotency. Nat Cell Biol. 2011;3:490-6.

10. Wu T, Pinto HB, Kamikawa YF, Donohoe ME. The BET family member BRD4 interacts with OCT4 and regulates pluripotency gene expression. Stem Cell Rep. 2015;:4:390-403.

11. Loewer S, Cabili MN, Guttman M, Loh YH, Thomas K, Park IH, et al. Large intergenic non-coding RNA-RoR modulates reprogramming of human induced pluripotent stem cells. Nat Genet. 2010;42:1113-7.

12. Boo K, Bhin J, Jeon Y, Kim J, Shin HJ, Park JE, et al. Pontin functions as an essential coactivator for Oct4-dependent lincRNA expression in mouse embryonic stem cells. Nat Commun. 2015;6:6810. 
13. Moran VA, Perera RJ, Khalil AM. Emerging functional and mechanistic paradigms of mammalian long non-coding RNAs. Nucleic Acids Res. 2012 40:6391-400.

14. St Laurent $G$, Wahlestedt C, Kapranov P. The landscape of long noncoding RNA classification. Trends Genet. 2015;31:239-51.

15. Gupta RA, Shah N, Wang KC, Kim J, Horlings HM, Wong DJ, et al. Long non-coding RNA HOTAIR reprograms chromatin state to promote cancer metastasis. Nature. 2010;464:1071-6.

16. Chakravarty D, Sboner A, Nair SS, Giannopoulou E, Li R, Hennig S, et al. The oestrogen receptor alpha-regulated IncRNA NEAT1 is a critical modulator of prostate cancer. Nat Commun. 2014;5:5383.

17. Tsai MC, Manor O, Wan Y, Mosammaparast N, Wang JK, Lan F, et al. Long noncoding RNA as modular scaffold of histone modification complexes. Science. 2010;329:689-93.

18. Li Z, Zhao X, Zhou Y, Liu Y, Zhou Q, Ye H, et al. The long non-coding RNA HOTTIP promotes progression and gemcitabine resistance by regulating HOXA13 in pancreatic cancer. J Transl Med. 2015;13:84.

19. Wang KC, Yang YW, Liu B, Sanyal A, Corces-Zimmerman R, Chen Y, et al. A long noncoding RNA maintains active chromatin to coordinate homeotic gene expression. Nature. 2011;472:120-4.

20. Niazi F, Valadkhan S. Computational analysis of functional long noncoding RNAs reveals lack of peptide-coding capacity and parallels with 3' UTRs. RNA. 2012:18:825-43.

21. Quek XC, Thomson DW, Maag JL, Bartonicek N, Signal B, Clark MB, et al. IncRNAdb v2.0: expanding the reference database for functional long noncoding RNAs. Nucleic Acids Res. 2015:43:D168-73.

22. Alheim K, Corness J, Samuelsson MK, Bladh LG, Murata T, Nilsson T, et al. Identification of a functional glucocorticoid response element in the promoter of the cyclin-dependent kinase inhibitor p57Kip2. J Mol Endocrinol. 2003;30:359-68

23. Cruickshanks HA, McBryan T, Nelson DM, Vanderkraats ND, Shah PP, van Tuyn J, et al. Senescent cells harbour features of the cancer epigenome. Nat Cell Biol. 2013;15:1495-506

24. Wang $X$, Li M, Wang Z, Han S, Tang X, Ge Y, et al. Silencing of long noncoding RNA MALAT1 by miR-101 and miR-217 inhibits proliferation, migration and invasion of esophageal squamous cell carcinoma cells. J Biol Chem. 2014;290:3925-35.

25. Wu W, Zhang S, Li X, Xue M, Cao S, Chen W. Ets-2 regulates cell apoptosis via the Akt pathway, through the regulation of urothelial cancer associated 1, a long non-coding RNA, in bladder cancer cells. PLoS One. 2013;8:e73920.

26. Li Y, Li Y, Chen W, He F, Tan Z, Zheng J, et al. NEAT expression is associated with tumor recurrence and unfavorable prognosis in colorectal cancer. Oncotarget. 2015;6:27641-50.

27. Pan $\sqcup$, Zhong TF, Tang RX, Li P, Dang YW, Huang SN, et al. Upregulation and clinicopathological significance of long non coding NEAT1 RNA in NSCLC tissues. Asian Pac J Cancer Prev. 2015;16:2851-5.

28. Sun C, Li S, Zhang F, Xi Y, Wang L, Bi Y, et al. Long non-coding RNA NEAT1 promotes non-small cell lung cancer progression through regulation of miR-377-3p-E2F3 pathway. Oncotarget. 2016; doi:10.18632/oncotarget.10108.

29. Choudhry H, Albukhari A, Morotti M, Haider S, Moralli D, Smythies J, et al. Tumor hypoxia induces nuclear paraspeckle formation through HIF-2a dependent transcriptional activation of NEAT1 leading to cancer cell survival. Oncogene. 2015;34:4482-90.

30. Lo PK, Zhang Y, Wolfson B, Gernapudi R, Yao Y, Duru N, et al. Dysregulation of the BRCA1/long non-coding RNA NEAT1 signaling axis contributes to breast tumorigenesis. Oncotarget. 2016; doi:10.18632/ oncotarget.11364.

31. Ji P, Diederichs S, Wang W, Böing S, Metzger R, Schneider PM, et al. MALAT-1, a novel noncoding RNA, and thymosin beta4 predict metastasis and survival in early-stage non-small cell lung cancer. Oncogene. 2003;22:8031-41.

32. Schmidt LH, Spieker T, Koschmieder S, Schäffers S, Humberg J, Jungen D, et al. The long noncoding MALAT-1 RNA indicates a poor prognosis in non-small cell lung cancer and induces migration and tumor growth. J Thorac Oncol. 2011;6:1984-92.

33. Gutschner T, Hämmerle M, Eissmann M, Hsu J, Kim Y, Hung G, et al. The noncoding RNA MALAT1 is a critical regulator of the metastasis phenotype of lung cancer cells. Cancer Res. 2013;73:1180-9.

34. Li S, Wang Q, Qiang Q, Shan H, Shi M, Chen B, et al. Sp1 mediated transcriptional regulation of MALAT1 plays a critical role in tumor. J Cancer Res Clin Oncol. 2015;141:1909-20.
35. Tano K, Mizuno R, Okada T, Rakwal R, Shibato J, Masuo Y, et al. MALAT-1 enhances cell motility of lung adenocarcinoma cells by influencing the expression of motilityrelated genes. FEBS Lett. 2010;584:4575-80.

36. Fan $Y$, Shen $B$, Tan $M, M u ~ X$, Qin $Y$, Zhang F, et al. TGF- $\beta$-induced upregulation of malat1 promotes bladder cancer metastasis by associating with suz12. Clin Cancer Res. 2014;20:1531-41.

37. Xu C, Yang M, Tian J, Wang X, Li Z. MALAT-1: a long non-coding RNA and its important 3' end functional motif in colorectal cancer metastasis. Int J Oncol. 2011;39:169-75.

38. Lai MC, Yang Z, Zhou L, Zhu QQ, Xie HY, Zhang F, et al. Long non-coding RNA MALAT-1 overexpression predicts tumor recurrence of hepatocellular carcinoma after liver transplantation. Med Oncol. 2012;29:1810-6.

39. Zhou X, Liu S, Cai G, Kong L, Zhang T, Ren Y, et al. Long non coding RNA MALAT1 promotes tumor growth and metastasis by inducing epithelial-Mesenchymal transition in oral squamous cell carcinoma. Sci Rep. 2015;5:15972.

40. Wang F, Ren S, Chen R, Lu J, Shi X, Zhu Y, et al. Development and prospective multicenter evaluation of the long noncoding RNA MALAT-1 as a diagnostic urinary biomarker for prostate cancer. Oncotarget. 2014;5: 11091-102.

41. Zhao $Y$, Yang $Y$, Trovik J, Sun $K$, Zhou L, Jiang P, et al. A novel wnt regulatory axis in endometrioid endometrial cancer. Cancer Res. 2014; 74:5103-17

\section{Submit your next manuscript to BioMed Central and we will help you at every step:}

- We accept pre-submission inquiries

- Our selector tool helps you to find the most relevant journal

- We provide round the clock customer support

- Convenient online submission

- Thorough peer review

- Inclusion in PubMed and all major indexing services

- Maximum visibility for your research

Submit your manuscript at www.biomedcentral.com/submit
) Biomed Central 